\title{
Für eine Geographie des (Post-)Politischen
}

Rezension zu Erik Swyngedouw (2018): Promises of the Political. Insurgent Cities in a Post-Political Environment. Cambridge/London: MIT Press.

Lucas Pohl

Abb. 1 Titel des Buches (Quelle: MIT Press)

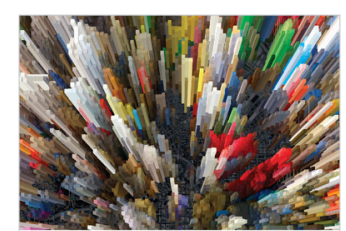

PROMISES OF THE POLITICAL INSURGENT CITIES IN A
POST-POLITCAL ENVIRONMENT ERIK SWYNGEDOUW

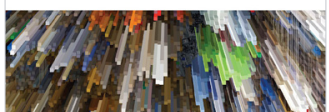

Erik Swyngedouw versammelt in seinem jüngsten Buch Promises of the Political: Insurgent Cities in a Post-Political Environment (2018) seine Arbeiten der vergangenen Dekade. Swyngedouw hat sich in der jüngeren Vergangenheit vor allem bezüglich seiner Ansätze zur Frage des Post-Politischen in die Debatten der kritischen Geographie und Stadtforschung eingeschrieben (für eine Auseinandersetzung in s u b \u r b a n, vgl. Michel/Roskamm 2013; Swyngedouw 2013). Seine wegweisenden, wenn auch kontrovers diskutierten Aufsätze zu den Möglichkeiten beziehungsweise Unmöglichkeiten des Politischen, die der Begriff, Post-Politik' vereint, werden in Promises of the Political zu einem konsistenten Ansatz ausgearbeitet und in ein übergreifendes Projekt gerahmt. Das Buch gliedert sich in drei Teile, wobei der erste Teil die theoretischen Grundlagen von Swyngedouws Ansatz zum Post-Politischen erläutert, der zweite Teil einen empirischen Blick auf gegenwärtige Formen post-politischer Praxis wirft und der dritte Teil schließlich die Möglichkeiten einer Rückkehr des Politischen in Betracht zieht.

\section{Eine kleine Geschichte der Post-Politik}

Swyngedouw beginnt den ersten Teil „Post-Democracy: Thinking (Post-) Politicization" (1-62) mit einer schlaglichtartigen Analyse der Transformationen des Staates, wie sie, ausgehend vom 18. Jahrhundert, unter anderem in den Arbeiten von Michel Foucault erörtert wurde. Im Anschluss an die staatstheoretischen Debatten der letzten Jahrzehnte konstatiert Swyngedouw die grundlegende Tendenz der Auflösung des Staates im Sinne einer eigenständigen beziehungsweise geschlossenen Sphäre der Politik in Abgrenzung etwa zum Markt als Sphäre der Ökonomie. Der vieldiskutierte Übergang von Government zu Governance und die damit verbundene Etablierung von Regierungstechniken jenseits beziehungsweise außerhalb des Staates sind aus Sicht Swyngedouws essentiell, um die aktuelle politische Situation als post-politische Situation zu verstehen. Prozesse der Privatisierung und Deregulierung führen zu einer Auslagerung staatlicher Funktionen, während das upscaling und downscaling von Governance, also die Übertragung 
politischer Belange an einerseits internationale und andererseits lokale Akteure, zu einer vertikalen Dezentralisierung des Regierens führen. Während ein Großteil der Governance-Prozesse mit dem Versprechen einer Ausweitung der Demokratie verbunden sind, skizziert Swyngedouw, wie die Re-Skalierung von Regierungstechniken im Gegenteil die Grundlage dafür bietet, dass Politik - in einem disruptiven und emanzipatorischen Sinne - zunehmend unmöglich wird. Wenn der Bereich des Politischen sich in Fragen des Managements, der Partizipation, der Legitimation und des Anrechts auf Teilhabe erschöpft, kommt es zu einer Einhegung und schließlich zur Auflösung dessen, was ,das Politische‘ im Kern auszeichnet. Die aktuelle (post-)politische Situation ist, so Swyngedouw, dadurch bestimmt, jeden Konflikt im Konsens zu ersticken beziehungsweise das, was nicht zu einem Konsens führt, in den Bereich des Unmöglichen zu verdrängen. Verdrängung zeichnet sich jedoch, wie Swyngedouw im Anschluss an den psychoanalytischen Verdrängungsbegriff mehrfach betont, dadurch aus, dass das Verdrängte „zurückkehrt“ (37). Keine noch so unschöne und unangenehme Angelegenheit kann auf ewig verdrängt werden. Um diesen Gedanken weiter zu verfolgen, widmet sich Swyngedouw der Differenzierung zwischen ,der Politik' (la politique) und ,dem Politischen' (le politique), wie sie vor allem im Kontext der französischen Philosophie eingeführt und über den Postfundamentalismus als konsistentes Theoriegebäude sowohl in der Soziologie (vgl. Marchart 2010) als auch in der Stadtforschung (vgl. Roskamm 2017) weiterentwickelt wurde. Dieser Ansatz ist für Swyngedouw deshalb von Bedeutung, weil er es in letzter Instanz gestattet, an der Existenz des Politischen trotz dessen gegenwärtiger Verdrängung festzuhalten. Auch wenn Swyngedouws Ansatz folglich nicht mit der Parole ,alles ist politisch“ vereinbar ist, da er davon ausgeht, dass nicht alle Formen des Protests in das Register des Politischen fallen (eine Ausführung erfolgt im Verlauf dieser Rezension), verfolgt Swyngedouw eine klare Vorstellung davon, was das Politische im Kern auszeichnet. Diese Vorstellung korreliert mit der folgenden Aussage von Oliver Marchart: „Nicht ,alles ist politisch“, sondern der Grund/Abgrund von allem ist das Politische.“ (2010: 364)

\section{Die post-politische Natur}

Bevor sich Swyngedouw im dritten Teil des Buches der Rückkehr des Politischen widmet, setzt er sich zunächst im zweiten Teil „Practices of PostPoliticization“ (63-125) mit zwei Schauplätzen auseinander, die er als zentral erachtet, um dem post-politischen Status quo empirisch nachzugehen: den Prozessen, Kämpfen und Konflikten rund um die Stadt und um die Natur. Im Folgenden werde ich mich aus Platzgründen auf Swyngedouws Aussagen zur Post-Politisierung der Natur beschränken, auch weil mein Eindruck ist, dass Swyngedouw hierauf den Schwerpunkt seiner Argumentation legt.

In den ersten zwei Unterkapiteln des zweiten Teils erörtert Swyngedouw die zentralen Argumente seiner bereits weitreichend rezipierten Analyse des politischen Umgangs mit dem Klimawandel. Im Anschluss an die philosophischen Arbeiten von Slavoj Žižek und Alain Badiou, die für Swyngedouws gesamtes Vorhaben zentrale Impulse liefern, aber auch mit Verweis auf Timothy Morton und Bruno Latour argumentiert Swyngedouw für eine 
radikale Verabschiedung des Naturbegriffs. Natur, im Sinne eines Gegensatzes zu Kultur, müsse demnach als ,leerer Signifikant v verstanden werden, der sich einer einheitlichen Bedeutungszuschreibung entzieht und nur dadurch Gewicht erhält, dass er die Struktur einer bestimmten sozialen Fantasie beflügelt. Es sei demnach kein Wunder, dass die Natur in unterschiedlichen geographischen und historischen Kontexten völlig unterschiedliche Assoziationen hervorrufe - von den romantisierten Naturvorstellungen des neunzehnten Jahrhunderts bis hin zu den mit Gefahr und Angst konnotierten Naturbezügen, die im Zusammenhang mit Infektionen, Waldbränden oder Überflutungen zum Ausdruck kommen. Swyngedouw warnt davor, in diesen unterschiedlichen Bezügen so etwas wie einen Grund der Natur zu suchen. Im Sinne des Postfundamentalismus müsse es eher darum gehen, sich der Grundlosigkeit von Natur bewusst zu werden und die vielfältigen MenschUmwelt-Assemblages anzuerkennen, die uns umgeben, um schließlich im zweiten Schritt der politischen Frage nachzugehen, in welchen sozionatürlichen Konfigurationen wir leben wollen. In den Debatten rund um Nachhaltigkeit sieht Swyngedouw eines der bahnbrechendsten Phantasmen, die einer solchen Politisierung von Natur im Weg stehen. Die Nachhaltigkeit belaufe sich demzufolge auf eine post-politische Fantasie, welche die Natur (anstelle der Religion) zum neuen Opium des Volkes werden lasse. Als Versprechen, uns vor der nahenden Apokalypse zu schützen, wird alles und jede*r dem Regime der Nachhaltigkeit unterworfen.

Im weiteren Verlauf seiner Argumentation setzt sich Swyngedouw vertiefender mit dem Narrativ vom Ende der Welt auseinander. Auch wenn die Faszination vom Weltuntergang eine lange Tradition im westlichen Glauben und Denken hat, verschiebt sich die tatsächliche Bedrohung des Planeten aktuell immer stärker ins politische Bewusstsein. Im Kontext des Klimawandels wird die Apokalypse zu einer immer bedeutenderen Komponente (post-)politischer Praxis. Die Konsequenzen globaler Erwärmung haben noch nie für derart viele Diskussionen gesorgt. Klimakonferenzen mutieren zu Großereignissen, vergleichbar mit den Olympischen Spielen, Klimaaktivist*innen werden zu ernstzunehmenden Akteuren im politischen Weltgeschehen und Wetterereignisse wie Tsunamis und Waldbrände werden zu düsteren Vorboten, die uns mit den Grenzen unseres eigenen Handelns konfrontieren.

Was die aktuellen Imaginationen vom Ende der Welt vereint, ist Swyngedouw zufolge die Vorstellung einer dystopischen Zukunft, welche die Menschheit als Ganze bedroht. Eine solche Perspektive folgt einer populistischen Logik, die wiederum mit einer starken Homogenisierung vollkommen unterschiedlicher Akteure einhergeht und darüber hinwegtäuscht, dass die Apokalypse für viele Menschen bereits längst zur Gegenwart geworden ist. Anstatt sich jedoch den bereits stattfindenden sozio-ökologischen Katastrophen in vielen Teilen der Welt zu widmen, rekurriert die populistische Imagination vom Ende der Welt darauf, die Apokalypse als steuerbares Krisenszenario zu verhandeln, in der es um jeden Preis darum geht, den gegenwärtigen Status quo ,für Alle zu erhalten. Swyngedouw zufolge ist es deshalb kein Zufall, dass die Konstruktion dystopischer Zukunftsvisionen oft von elitären Positionen beflügelt wird (Al Gore als ein prominentes Beispiel), weil der Erhalt des Status quo in erster Linie von solchen Positionen aus wünschenswert und notwendig erscheint. 
Dagegen plädiert Swyngedouw dafür, die Apokalypse im Hier und Jetzt als eine „ungleiche Apokalypse“ (97) zu verstehen, die nicht von der Menschheit als Ganzer, sondern von denen getragen wird, die in der Welt, die vermeintlich auf dem Spiel steht, ohnehin keinen Platz haben.

\section{Die Rückkehr des Politischen}

Im dritten und letzten Teil des Buches „Specters of the Political“ (127-169) widmet sich Swyngedouw eingehender der Frage, wie es zu einer Re-Politisierung in Zeiten des Post-Politischen kommen kann. Im Anschluss an die Aufstände, die sich in den vergangenen Jahren in unterschiedlichen geographischen Kontexten zugetragen haben, argumentiert Swyngedouw für eine neue Protestform, die sich von tradierten Formen städtischer Bewegungen unterscheidet und eine radikale Demokratisierung des bestehenden Systems einfordert. Die Proteste in Städten wie Istanbul, Kairo, Tunis, Athen, Madrid, New York, Tel Aviv, Chicago, London, Berlin, Thessaloniki, Santiago, Stockholm, Hong Kong, Cape Town, Barcelona, Montreal und anderen ähneln sich demnach darin, dass hier diejenigen eine Stimme einfordern, die im gegenwärtigen (demokratischen) Konsens keine haben. Im Anschluss an Jacques Rancière (2002) und dessen Kategorie der „Anteillosen“ sowie Badious (2013) Figur der „Inexistenten“ spricht Swyngedouw von einer gespenstischen Rückkehr des Politischen, in der eine vermeintlich minoritäre beziehungsweise unsichtbare Gruppe der Bevölkerung eine Sichtbarkeit erlangt, um zu einem Stellvertreter für die Gesamtheit der Bevölkerung zu werden. Vor diesem Hintergrund warnt Swyngedouw davor, das Politische im Sinne von le politique - in den Mikropolitiken lokaler Kämpfe zu suchen. Er plädiert im Gegenzug für einen starken Begriff des Politischen, wonach nur solche Kämpfe wirklich politisch sind, welche die Grundfesten des gesamten sozialen Raums infrage stellen.

Es ist vor allem der letzte Teil des Buches, in dem ersichtlich wird, mit welcher Vehemenz sich Swyngedouw gegen die Akzeptanz einer post-politischen Gegenwart positioniert. Entgegen der Annahme, Swyngedouw kreiere die Vorstellung einer „omnipotenten“ Ordnung des Post-Politischen (Beveridge/Koch 2017: 7), legt Promises of the Political einen Zugang, der die „Impotenz“ dieser Ordnung konstatiert (167), um dadurch zu einer radikalen Re-Imagination des Politischen zu gelangen. Radikal ist Swyngedouw nicht nur, weil er den gewaltvollen Aufständen der jüngeren Vergangenheit in Ländern wie Frankreich, Griechenland und Italien verständnisvoll gegenübersteht, sondern vor allem deshalb, weil er es gestattet, einen offenkundig revolutionstheoretischen Ansatz in die Geographie zu importieren. Im Anschluss an Badiou argumentiert Swyngedouw, dass wahre Politik darauf gründet, neue Fiktionen hervorzubringen. Vor diesem Hintergrund widmet sich Swyngedouw im letzten Unterkapitel des Buches der Idee des Kommunismus, die er - erneut im Anschluss an Badiou (2011) - als zugleich randständigste und radikalste Fiktion im Register des Politischen verhandelt.

Insofern die kommunistische Hypothese zugleich das Prinzip der Gleichheit als auch das Prinzip der demokratischen Selbstverwaltung auf den Plan ruft, steht sie als Name für die Denkbarkeit und Möglichkeit einer anderen Welt. Ein solcher Name ist aus Sicht Swyngedouws gerade deshalb nötig, 
da die Demokratie ihre Funktion als Bezeichnung für den Horizont sozialer Praxis eingebüßt hat. Insofern Demokratie zunehmend mit individueller (Wahl-)Freiheit und nicht mit einem universellen Recht auf Gleichheit assoziiert wird, verliert sie ihren emanzipatorischen Anspruch. Hinzu kommt, dass die zunehmende Privatisierung des Staates und die Finanzialisierung jeglicher Lebensbereiche dazu führen, dass nichts und niemand vor den Regeln des Marktes gefeit ist. Die verallgemeinerte Stimmung der Angst (vor Globalisierung, Terrorismus, Klimawandel, Migration etc.) und der verbreitete Anstieg von Nationalismus, Rassismus und Xenophobie sind ein Resultat dieser Entwicklung. Der Kommunismus wiederum erlaube einen radikalen Bruch mit diesen Entwicklungen, insofern er zu einem treuen Glauben und einem bedingungslosen Aufruf nach Gleichheit und Freiheit für Alle auffordert. Schließlich verweist Swyngedouw darauf, dass die Angst vor dem (erneuten) Scheitern des Kommunismus uns gegenwärtig mit einer allgemeinen Alternativlosigkeit konfrontiert, die es nötig werden lässt, sich darauf zu beschränken, den gegenwärtigen Ist-Zustand bestmöglich aufrechtzuerhalten. Im Gegensatz hierzu plädiert Swyngedouw abschließend dafür, die fruchtbaren Impulse aus der Theorie ernst zu nehmen und das Risiko des Scheiterns in Kauf zu nehmen, um eine kommunistische Geographie zu ermöglichen, die den Status quo zu überwinden versucht.

\section{Auf zu neuen Ufern}

Der Ansatz, den Swyngedouw in Promises of the Political entwickelt, liest sich als eine Doppelbewegung, in der die soziale Realität zunächst minutiös erfasst und Stück für Stück seziert wird, um dann in einem zweiten Schritt auf die Inkonsistenzen und Risse in dieser Realität hinzuweisen. Dabei wird in Analogie zur Psychoanalyse darauf insistiert, dass Fantasien beziehungsweise Phantasmen einen entscheidenden Einfluss darauf haben, wie sich unsere soziale Realität zusammensetzt. Die Fantasien ernst zu nehmen, die sich beispielsweise entlang der politischen Debatten rund um Nachhaltigkeit entspinnen, ist sicherlich eine der größten Stärken von Swyngedouws Ansatz. Dadurch wird es möglich, eine neue ideologiekritische Perspektive in die Geographie und Stadtforschung einzuführen, die sich von der marxistischen Standardvorstellung von Ideologie als, falschem Bewusstsein“ verabschiedet. Inspiriert von den frühen Arbeiten Žižeks zielt Swyngedouw darauf, Ideologie nicht als Verzerrung der Realität, sondern als notwendige Voraussetzung für die Entstehung von Realität zu verstehen: „Die Funktion der Ideologie besteht nicht darin, uns einen Fluchtpunkt aus unserer Realität anzubieten, sondern uns die soziale Realität selbst als Flucht vor einem traumatischen, realen Kern anzubieten. “(Žižek 1989: 45, Übers. d. A.) Swyngedouw schließt unmittelbar hieran an, insofern er den ideologischen Realitätskonstruktionen der (Post-)Politik nachgeht, um diese hinsichtlich ihres verdrängten Kerns - dem Politischen - zu befragen. Ein solches Vorgehen nimmt es sich somit zum Ziel, das soziale Gefüge von innen heraus zu erschüttern, ihm gewissermaßen die ,Grundlagen' zu entziehen und aufzuzeigen, auf welch wackligen Beinen unsere alltägliche Realität steht.

Promises of the Political legt damit den Grundstein für eine tiefergehende Erörterung der Frage danach, wie Geographie und Stadtforschung nicht nur 
dazu beitragen, die gegenwärtige Situation als (post-)politische Situation besser zu verstehen, sondern zugleich über diese Situation hinauszugehen. Swyngedouw beschränkt sich keineswegs darauf, einen post-politischen Fatalismus zu proklamieren, in dem nichts mehr wirklich politisch ist. Auch wenn er in der Vergangenheit bereits des Öfteren eines solchen Fatalismus bezichtigt wurde (vgl. Beveridge/Koch 2017), geht es ihm vielmehr darum, die inhärenten Brüche des Post-Politischen, das „Unbehagen im Post-Politischen“ (Wilson/Swyngedouw 2014), freizulegen, um darin eine Rückkehr des Politischen zu situieren. Dadurch gelingt es Swyngedouw, das Feld der Geographie und Stadtforschung in einer radikalen Weise zu öffnen. Fragen der Revolution und des Kommunismus, denen man gegenwärtig sowohl innerhalb als auch außerhalb der Wissenschaft weitestgehend mit ironischer Distanz gegenübersteht, werden hier zu ernsthaften Bestandteilen der kritischen Geographie und Stadtforschung erhoben. Der Bereich des Sagbaren verschiebt sich - erfrischender Weise mal nicht im Sinne der neuen Rechten.

Die Publikation dieses Beitrags wurde durch die Deutsche Forschungsgemeinschaft (DFG) und den Open Access-Fonds der Humboldt-Universität zu Berlin ermöglicht.

\section{Autor_innen}

Lucas Pohl ist Humangeograph und beschäftigt sich mit der Vermittlung von Geographie, Philosophie und Psychoanalyse in Bezug auf Fragen der Stadt- und Raumtheorie, gebauten Umwelt und politischen Praxis.

lucas.pohl@geo.hu-berlin.de

\section{Literatur}

Badiou, Alain (2011): Die kommunistische Hypothese. Berlin: Merve.

Badiou, Alain (2013): Das Erwachen der Geschichte. Wien: Passagen.

Beveridge, Ross / Koch, Philippe (2017): The post-political trap? Reflections on politics, agency and the city. In: Urban Studies 54/1, 31-43.

Marchart, Oliver (2010): Die politische Differenz. Zum Denken des Politischen bei Nancy, Lefort, Badiou, Laclau und Agamben. Berlin: Suhrkamp.

Michel, Boris / Roskamm, Nikolai (2013): Einführung. Die ,postpolitische Stadt‘. In: sub\ urban. zeitschrift für kritische stadtforschung 1/2, 9-16.

Rancière, Jacques (2002): Das Unvernehmen: Politik und Philosophie. Frankfurt am Main: Suhrkamp.

Roskamm, Nikolai (2017): Die unbesetzte Stadt. Postfundamentalistisches Denken und das urbanistische Feld. Basel: Birkhäuser.

Swyngedouw, Erik (2013): Die postpolitische Stadt. In: sub \urban. zeitschrift für kritische stadtforschung 1/2, 141-158.

Swyngedouw, Erik (2018): Promises of the Political. Insurgent Cities in a Post-Political Environment. Cambridge: MIT Press.

Wilson, Japhy / Swyngedouw, Erik (2014) (Hg.): The Post-Political and Its Discontents: Spaces of Depoliticization, Spectres of Radical Politics. Edinburgh: Edinburgh University Press.

Žižek, Slavoj (1989): The Sublime Object of Ideology. London/New York: Verso. 